\title{
Internes Qualitätsmanagement und externe Qualitätstransparenz in der operativen Gynäkologie
}

\section{H.-K. Selbmann}

M. Geraedts

\section{Einleitung}

In der seit Anfang des Jahres 2004 gültigen Fassung des §135a des 5. Sozialgesetzbuchs wird festgestellt, dass „die Leistungserbringer zur Sicherung und Weiterentwicklung der Qualität der von ihnen erbrachten Leistungen verpflichtet sind." Zudem verpflichtet der § 135a alle Leistungsanbieter - also auch die Abteilungen für operative Gynäkologie und die niedergelassenen Gynäkologen

1. sich an einrichtungsübergreifenden Maßnahmen der Qualitätssicherung zu beteiligen, die insbesondere zum Ziel haben, die Ergebnisqualität zu verbessern und

2. einrichtungsintern ein Qualitätsmanagement einzuführen und weiterzuentwickeln.

Darüber hinaus verpflichtet das Sozialgesetz im §137 SGB V Abs. 1 alle Krankenhäuser, ab 2005 zweijährlich in einem Qualitätsbericht strukturiert über den Stand der Qualitätssicherung zu berichten. Dabei ist u.a. darzustellen, inwieweit Strukturanforderungen an die Qualität erfüllt werden, die ab 2004 vom neu geschaffenen Gemeinsamen Bundesausschuss beschlossen werden. $\mathrm{Zu}$ diesen Strukturanforderungen gehören unter anderem auch Mindestmengen für spezielle Leistungen - die Gynäkologie ist davon in 2004 noch nicht betroffen - pro Arzt oder Krankenhaus.

Mit externen Vergleichen hat die operative Gynäkologie in den Pilotstudien von 1984 und von 1992-96 ausreichend Erfahrungen sammeln können und funktionstüchtige Verfahren erarbeitet (Eichhorn et al., 1989; Geraedts et al., 1998). Im Jahr 1999 hatte der Gesetzgeber die Grenzen der externen Vergleiche bezüglich der Qualitätsverbesserung erkannt und die Verpflichtung zum internen Qualitätsmanagement in das Gesetz mit aufgenommen.

Neben den genannten gesetzlich verordneten Maßnahmen zur Qualitätssicherung und -verbesserung gibt es eine Reihe weiterer Maßnahmen, die sich langsam in Deutschland ausbreiten bzw. im Ausland bereits Platz gegriffen haben. Es sind dies das Qualitätsmanagement für Versorgungsnetzwerke, das Peer Reviewing, die Zertifizierung von Krankenhäusern und Abteilungen und die intensive Patienteninformierung. Sowohl die bereits existierenden als auch die zurzeit diskutierten bzw. sich entwickelnden Ansätze zum Qualitätsmanagement in der operativen Gynäkologie werden im Folgenden der Reihe nach behandelt.

\section{Internes Qualitätsmanagement füir gynälkologische Versorgungseinrichtungen}

Nach der ISO EN DIN-Norm 9000:2000 umfasst das Qualitätsmanagement (QM) alle aufeinander abgestimmten Tätigkeiten zur Leitung und Lenkung einer Organisation, mit denen die Qualitätspolitik und die Qualitätsziele festgelegt sowie diese durch Qualitätsplanung, Qualitätslenkung, Qualitätssicherung und Qualitätsverbesserung verwirklicht werden. Übertragen auf gynäkologische Abteilungen und Praxen heißt das, dass sie sich als Unternehmen verstehen sollen, die explizite Qualitätsziele haben und diese durch Methoden der Planung, Kontrolle, Sicherung und Verbesserung zu erreichen versuchen. 
Wesentlichstes Element des internen Qualitätsmanagements ist der kontinuierliche Verbesserungsprozess (KVP), der mit der Erkennung und Kommunikation von mutmaßlichen Qualitätsproblemen beginnt. Nach der Problemverifizierung und -analyse und dem Auffinden von Problemlösungen folgt der wohl schwierigste Schritt dieses Prozesses, die Umsetzung der Lösungen in den Versorgungsalltag. Die beiden Schritte „Evaluation der umgesetzten Problemlösungen“ und „Sicherstellung, dass die Probleme nicht erneut auftreten“ schließen den Kreis (in der QM-Literatur auch PDCA-Zyklus - von Plan, Do, Check, Act - genannt) und man kann sich neuen Problemen zuwenden. Da in der Krankenversorgung immer etwas zu verbessern ist - dies liegt u.a. auch an den begrenzten Ressourcen - ist es in der Regel nicht schwer, im Versorgungsalltag neue Probleme für den KVP zu finden.

Eine gynäkologische Einrichtung mit einem funktionierenden internen Qualitätsmanagement zeichnet sich u.a. durch folgende Charakteristika aus:

- eine engagierte oberste Leitungsebene, die dem Unternehmen ein Leitbild gibt, Qualitätsziele formuliert, die Mitarbeiter zur Qualitätsverbesserung motiviert und dabei selbst Vorbild ist,

- geeignete Qualitätsmanagementstrukturen wie eine Qualitätskonferenz und mindestens einen geschulten Qualitätskoordinator (nicht Qualitätsbeauftragten). Weiter müssen die Ressourcen für problemorientierte Qualitätszirkel vorhanden sein,

- die richtigen Mitarbeiter, die qualitätsbewusst, kundenorientiert, teamfähig, veränderungsbereit und fachlich kompetent die Qualität planen, prüfen, sichern und ggf. verbessern. Die Schaffung der psychosozialen Kompetenz bei den Mitarbeitern ist sicher das schwierigste und langwierigste Unterfangen beim Aufbau eines internen Qualitätsmanagements,

- ein regelmäßiger Qualitätsbericht, mit dem die erreichte Qualität nach innen transparent gemacht und neue Qualitätsziele kommuniziert werden können. Der neue gesetzlich geforderte Qualitätsbericht kann diese Aufgaben nicht erfüllen,

- etablierte Problemerkennungsverfahren wie die Messung von Qualitätsindikatoren oder die Sammlung von Informationen über unerwünschte Ereignisse. Gerade Letzteres benötigt in der Abteilung eine Qualitätskultur, in der jede Schwachstelle als eine Möglichkeit zur Verbesserung verstanden wird. Solange das auf Schwachstellen aufmerksam Machen als Nestbeschmutzung verstanden und z. B. mit Nicht-Vertragsverlängerung geahndet wird, solange wird das interne Qualitätsmanagement einer Abteilung nicht funktionieren,

- Beherrschung der QM-Techniken (Problemanalyse, Priorisierung, Arbeit in multiprofessionellen Gruppen, Motivierung etc.) durch die Leitungsebene und die Mitarbeiter. Diese Techniken lassen sich erlernen. Die QM-Kurse der Landesärztekammern nach dem Curriculum Qualitätssicherung bieten seit 1997 beim Lernen Hilfestellung,

- geeignete qualitätssichernde Verfahren wie Hausleitlinien, hierarchische Entscheidungsverfahren, Morbiditäts- und Mortalitätskonferenzen etc.,

- Fähigkeit zur Darlegung der Qualität und des Qualitätsmanagement nach außen, um die Kunden - in der Hauptsache die Patienten, ihre Angehörigen, die mitbehandelnden Ärzte und die kooperierenden Krankenhäuser - über die zu erwartende Qualität informieren zu können.
Die Exzellenz einer gynäkologischen Einrichtung kann man z.B. mithilfe des 1999 überarbeiteten Verfahrens der European Foundation for Quality Management (EFQM) messen und bewerten. Die neun EFQM-Kriterien Führung, Politik und Strategie, Mitarbeiter, Partnerschaften und Ressourcen, Prozesse, kunden-, mitarbeiter- und gesellschaftsbezogene Ergebnisse sowie wichtige Ergebnisse der Abteilung bei den Schlüsselleistungen helfen dabei, nichts an Unternehmensexzellenz zu übersehen. Die Beteiligung erfahrener externer EFQM-Assessoren kann sinnvoll sein, um die Objektivität bei der Beobachtung der eigenen Qualität zu erhalten.

\section{Qualitätsmanagement für gynäkologische Versorgungsnetzwerke}

Wie für das interne Qualitätsmanagement von gynäkologischen Einrichtungen gilt auch für die so genannte „integrierte Versorgung“ ( $§ 140 a-d$ SGB V), dass die Qualität gesichert sein muss (Definition der integrierten Versorgung: Leistungssektoren übergreifende- oder interdisziplinär-fachübergreifende Versorgung). Solche integrierten Versorgungsformen können auch im Rahmen von Modellvorhaben oder Strukturverträgen in Form vernetzter Praxen (§63-65, §73a SGB V), bei strukturierten Behandlungsprogrammen für chronisch Kranke ( $\$ 137 \mathrm{fSGB} V$ ) und in von verschiedenen Leistungsanbietern gemeinsam betriebenen Brustzentren zum Einsatz kommen. Das grundsätzliche Ziel der integrierten Versorgung sollte sein, die Versorgungsqualität zu verbessern, indem vorhandene Koordinationsprobleme zwischen den verschiedenen Versorgungssektoren oder Disziplinen überwunden und dabei auch Wirtschaftlichkeitsreserven z. B. durch Unterlassung von Wiederholungsuntersuchungen ausgeschöpft werden. Im Kern sollen vertragliche Beziehungen zwischen den Leistungserbringern verschiedener Sektoren z. B. zwischen niedergelassenen Gynäkologen, Krankenhäusern, Rehabilitationseinrichtungen oder anderen ärztlichen (z. B. Laborärzte, Radiologen) bzw. nicht ärztlichen Leistungserbringern (z. B. Physiotherapeuten) dafür sorgen, dass Patienten mit hoher Kontinuität und Qualität wirtschaftlich versorgt werden.

Solange Maßnahmen wie etwa Leitlinien für Ein- und Überweisungen mit Absprache der vor- oder nachstationär zu erbringenden Leistungen von Netzwerken eingeführt werden, sind vor allem positive Effekte auf die Qualität der Versorgung zu erwarten. Werden jedoch vertragliche Beziehungen in Form von Einkaufsmodellen beschlossen - eine Krankenkasse kauft die für ihre Versicherten notwendigen ambulanten und stationären Leistungen zu vorab festgelegten Preisen bei ausgewählten Leistungserbringern ein - dann können sie sich durch falsch gesetzte finanzielle Anreize auch negativ auf die Qualität der Versorgung auswirken.

Genau deshalb will der Gesetzgeber die Betreiber von Versorgungsnetzwerken, die zumeist auch die wirtschaftliche Verantwortung für die medizinische Versorgung von Patientengruppen übernehmen, zur Qualitätssicherung verpflichten. Das „Wie“ des Qualitätsmanagements wird dabei den Versorgungsnetzwerken überlassen.

Auf der Suche nach Beispielen für Aktivitäten und Systeme für das Qualitätsmanagement von Versorgungsnetzwerken fällt ins- 
besondere die amerikanische Akkreditierungsorganisation für Managed Care Organisationen (MCO) ins Auge, das National Committee for Quality Assurance (NCQA, 1998). So verlangt das NCQA für eine Akkreditierung, dass die MCO folgende Kriterien im Bereich des Qualitätsmanagement (QM) und der Qualitätsverbesserung (QV) erfüllen. Diese Kriterienliste könnte auch in der gynäkologischen Versorgung Deutschlands als Vorbild für Versorgungsnetzwerke dienen. Zum Teil spiegeln sich diese Kriterien bereits in der Definition des Landes Nordrhein-Westfalen für Brustzentren wider:

1. QM-Programm: schriftliche Darlegung von Strukturen, Prozessen und Verantwortlichkeiten; Jahresarbeitsplan; angemessene Ressourcen für QV.

2. Aktiver Programm-Betrieb: protokollarischer Nachweis von QV-Aktivitäten.

3. Verträge mit Leistungserbringern: Offenlegung der Vertragsklauseln zur Kooperation der Ärzte, Krankenhäuser und anderer medizinischer Leistungserbringer im Bereich QM.

4. Erreichbarkeit von medizinischen Leistungserbringern: Nachweis einer ausreichenden Anzahl und einer geeigneten geographischen Verteilung von medizinischen Leistungserbringern.

5. Zugang zu Leistungen: Definition von Kriterien zur Verfügbarkeit und Zeitigkeit von Arztterminen, Notfallbehandlungen und Telefonservice-Leistungen.

6. Mitglieder-/Patientenzufriedenheit: Durchführung valider Mitglieder-/Patientenbefragungen und von Beschwerdeanalysen; Information der Leistungserbringer über die Ergebnisse.

7. Versorgungsmanagement-Systeme: Einführung und Wirksamkeitsanalysen von Disease-Management-Programmen für Patienten mit chronischen Erkrankungen.

8. Leitlinien: Anpassung, Verbreitung und Wirksamkeitsevaluierung evidenzbasierter Leitlinien.

9. Umfang und Inhalt klinischer QV-Aktivitäten: Überwachung und Evaluierung von mindestens drei klinischen Leistungsbereichen, die insbesondere auf die Über-/Unterversorgung, Kontinuität und Kooperation der Versorgung zu fokussieren sind.

10. Messung klinischer Leistungen: Durchführung valider und reliabler Datenerhebungen, -analysen und -bewertungen in mindestens drei klinischen Leistungsfeldern.

11. Intervention und Langzeitbeobachtung klinischer Leistungen: Nachweis der Intervention bei prioritären Problemen und (Langzeit-)Messung der Wirksamkeit der Interventionen.

12. Effektivität des QV-Programms: Nachweis der Wirksamkeit des QV-Programms durch Nachweis von Verbesserungen bei klinischen- und Serviceleistungen.

Zusammengefasst kommt es darauf an, dass Versorgungsnetzwerke zunächst Strukturen und Prozesse implementieren, die der kontinuierlichen Qualitätsverbesserung der Versorgung dienen. Anschließend ist es wichtig, die Effektivität der eingeführten Maßnahmen und die Funktionalität des Versorgungsnetzes empirisch zu belegen.

Zwischen dem Qualitätsmanagement von Versorgungseinrichtungen und dem von Versorgungsnetzwerken bestehen zwei wesentliche Unterschiede: Erstens geht bei Letzteren die Perspekti- ve bei der Messung der Ergebnisse von Versorgungsleistungen über die Kurzzeitergebnisse hinaus und umfasst die Langzeitergebnisse innerhalb eines Versorgungsnetzwerks. Zweitens verschiebt sich der Fokus des Qualitätsmanagements von einzelnen Leistungserbringern auf die Gesamtheit der an der Versorgung bestimmter Patientengruppen Beteiligten. Sind diese regional organisiert und beispielsweise alle in einer Region niedergelassenen Gynäkologen mitsamt den in der Region vorhandenen stationären gynäkologischen Abteilungen eingebunden, dann zeigt sich deutlich der Vorteil eines solchen bevölkerungsorientierten Qualitätsmanagements in Versorgungsnetzwerken: Betrachtet man etwa den Qualitätsindikator „Frequenz von Adnexoperationen ohne histopathologischen Befund“ nur in Krankenhausabteilungen, dann könnte die ausführende Klinik argumentieren, dass ihre hohe Frequenz zum Teil deshalb zustande kommt, weil sie auf die Operationswünsche eingeht, die zwischen einweisenden Ärzten und Patientinnen bereits abgesprochen waren. Bei der regionalen Betrachtung dieses Indikators würden alle in den Prozess der Indikationsstellung zur Operation Involvierten in die Qualitätsbeurteilung einbezogen. Gemeinsam tragen dann die Leistungserbringer die Verantwortung dafür, dass möglichst alle Patientinnen mit wahrscheinlich benignen Adnextumoren der Leitlinie entsprechend konservativ vorbehandelt zur Operation kommen. Eine solche bevölkerungsorientierte Qualitätsbewertung erzwingt förmlich die Kommunikation der Leistungserbringer sowie die Versorgungsintegration zum Wohl der Patientinnen. Hiermit ließe sich eine echte Qualitätsverbesserung für die Patientinnen auf der Grundlage von Qualitätsmanagement-Maßnahmen innerhalb von Versorgungsnetzwerken erzielen.

\section{Peer Reviewing in der Gynälkologie}

Eine in den Niederlanden sehr erfolgreiche Maßnahme zur externen Qualitätssicherung ist das Peer Reviewing - das so genannte VISITATIE-Programm der Nationalen Organisation für Qualitätssicherung (CBO). Fachkollegen (Peers) beurteilen dabei andere Fachkollegen, ein Verfahren, das in Deutschland bei der wissenschaftlichen Beurteilung von Sonderforschungsbereichen oder Klinischen Forschergruppen nicht unbekannt ist. Für nahezu alle Fächer - einschließlich der Gynäkologie - gibt es in den Niederlanden ausgearbeitete Vorgehensweisen und geschulte Peers, die die ausgefüllten Fragebogen studieren und sich bei einem strukturierten eintägigen Besuch vor Ort ein Bild von der Qualität der besuchten Einrichtung machen (Weert 2000). Bei den alle fünf Jahre vorgesehenen Besuchen wird zwar auch auf die vorhandene Strukturqualität (technische Ausstattung, räumliche Anordnungen, Zahl und Fortbildungsstand der Mitarbeiter etc.) geachtet, im Vordergrund stehen aber die Kernprozesse des ärztlichen und pflegerischen Handelns einschließlich der klinischen Dokumentation. Fragen nach den verwendeten Leitlinien, der Ablauforganisation, der Kooperation mit anderen Abteilungen und Funktionsbereichen, dem Wissen um die eigene Ergebnisqualität (z.B. Komplikations- und Infektionsstatistiken, Patientenzufriedenheit), der Qualität der Krankenblattführung und des Arztbriefes und den eingesetzten Maßnahmen zur Qualitätsförderung haben die besuchten Abteilungen zu beantworten. Nach einem Abschlussgespräch vor Ort werden die Ergebnisse dann in einem Besuchsbericht ggf. einschließlich konkreter Empfehlungen der Peers für Qualitätsverbesserungen festgehal- 
ten. Die hohe Akzeptanz des Peer Reviewing ist sicher darin begründet, dass erstens das Verfahren freiwillig und damit Vertrauenssache ist und zweitens die Peers nicht als Kontrolleure sondern als erfahrene Kollegen in die Abteilungen kommen und auch ihrerseits einen Nutzen davontragen. Auf Grund der Fokussierung auf die fachärztlichen Leistungen und der erreichbaren Bewertungstiefe ist das Peer Reviewing eine hervorragende Ergänzung zu anderen QM-Verfahren.

In Deutschland haben Pneumologen und Kardiologen die längsten Erfahrungen mit dem Peer Reviewing. Das Bundesministerium für Gesundheit förderte bis 2000 ein Projekt der kommunalen Kliniken Bremens, Hamburgs und Münchens zum Peer Reviewing in den Fächern Chirurgie, Gynäkologie/Geburtshilfe, Innere Medizin und Neurologie (Blum et al., 2002). Wohl wegen der organisatorischen Probleme und auch den damit verbundenen Kosten wurde jedoch bisher von einer Ausbreitung auf Deutschland abgesehen.

Im Rahmen der Zertifizierung von Brustzentren durch die Deutsche Krebsgesellschaft und die Deutsche Gesellschaft für Senologie finden ebenfalls Begehungen vor Ort statt, das einem Peer Reviewing ähnelt.

\section{Zertifizierung von Krankenhäusern und Abteilungen}

International wie national ist die Zertifizierung von Krankenhäusern einer der großen Hoffnungsträger der Gesundheitswesen und zwar nicht erst seit die Industrie 1987 damit begonnen hat. Die erste Zertifizierung eines deutschen Krankenhauses nach dem aus der Industrie kommenden ISO 9001-Modell (ISO: International Organisation for Standardisation) erfolgte 1995. Bereits 1996 hatten die Bundesärztekammer, die Deutsche Krankenhausgesellschaft und der VdAK jedoch darauf aufmerksam gemacht, dass die ISO-Zertifizierung kein geeignetes Instrument zur Qualitätsdarlegung für Krankenhäuser sein kann.

In den USA waren es die Colleges der Chirurgen und der Internisten, die amerikanische Krankenhausgesellschaft und andere Organisationen, die 1951 durch die Gründung der Joint Commission on Accreditation of Hospitals (später JCAHO) den Grundstein zu einem Akkreditierungsprogramm legten, bei dem heute ca. 19.000 Einrichtungen des Gesundheitswesens freiwillig zertifiziert (die US-Amerikaner verwenden dafür das Wort akkreditiert) sind. Ihren Spuren und Methoden folgten schon sehr früh Canada (1958) und zwanzig Jahre später Australien (1974). Seit dem Jahr 2000 existiert eine gegenüber dem JCAHO-Katalog verallgemeinerte, internationale Version des US-amerikanischen Zertifizierungsverfahrens, das von der Joint Commission International (JCI) auf dem Markt angeboten wird. Fünf deutsche Kliniken mit ihren Frauenkliniken verfügten in 2004 über das Zertifikat der JCI (www.jcrinc.com/international).

In Deutschland folgt die „Kooperation für Transparenz und Qualität (KTQ)“, die von der Bundesärztekammer, der Deutschen Krankenhausgesellschaft, dem Deutschen Pflegerat und den Spitzenverbänden der Gesetzlichen Krankenversicherungen getragen wird, und ihre konfessionelle Variante „proCumCert“ den großen internationalen Erfahrungen. Im Juni 2002 wurde das erste Krankenhaus zertifiziert. Bis Ende 2004 hatten sich bereits 129 Krankenhäuser - einschließlich ihrer gynäkologischen Abteilungen - zertifizieren lassen. Ihre Qualitätsberichte wurden als Grundvoraussetzung für das Erlangen des Zertifikats über das Internet der Allgemeinheit zugänglich gemacht (www. ktq.de).

Die Intention der genuin-medizinischen Zertifizierungsverfahren ist die nachvollziehbare Darlegung qualitativ guter Leistungen der Versorgungseinrichtungen: der hohen Qualität ihrer Leistungen und ihres Qualitätsmanagements. Ein abgestimmter Katalog an Standards und Kriterien für gute Versorgungsqualität dient dabei sowohl als Maßstab für die aktuelle Einschätzung als auch als Vorbild für weitere Qualitätsverbesserungen. Die Standards gelten für alle medizinischen Fachgebiete. Dieselbe Idee für den niedergelassenen Bereich verfolgt auch das in der Entwicklung befindliche Modell QEP (Qualität und Entwicklung in Praxen) der Kassenärztlichen Bundesvereinigung (www.kbv.de/ qm/qualitaet_index.htm).

Die genuin-medizinischen Zertifizierungsverfahren unterscheiden sich von der ISO-Zertifizierung u. a. dadurch, dass sie die Versorgung der Patienten von der Aufnahme bis zur Betreuung nach der Entlassung in den Mittelpunkt stellen. Dem Hauptbereich der Patientenversorgung nachgeordnet sind die fünf Bereiche Leitung, Management und Führung des Krankenhauses, Personal-Management und -Entwicklung, Informationsmanagement und Management der Versorgungsumwelt inkl. Sicherheit und Qualitätsverbesserung.

In allen sechs Bereichen verfügen die Verfahren über detaillierte Vorstellungen von guter Qualität (so genannte Standards). Diese Standards enthalten die Beschreibung der zu fordernden guten Prozesse und Ergebnisse. An diesen Standards müssen oder können sich dann die einzelnen Krankenhäuser messen (Selbstbewertung) oder messen lassen (Fremdbewertung). Bei der Bewertung wird festgestellt, in wieweit die eingesetzten Prozesse zur Zielerreichung geeignet sind und die Ziele erreicht wurden. Wurden sie nicht erreicht, können die Mitarbeiter der Einrichtung unschwer den Standards entnehmen, wie sie ihre Qualität verbessern können (Glück und Selbmann, 2000). Die Zertifikate der genuin-medizinischen Verfahren gelten immer für die gesamte Einrichtung, während nach dem ISO-Verfahren auch nur einzelne Abteilungen einer Einrichtung zertifiziert werden können.

Die Zertifizierung von Brustzentren mit ihren Anforderungen an eine gute Struktur- und Prozessqualität hat in den letzten 3 Jahren erheblich an Bedeutung gewonnen. In Nordrhein-Westfalen gibt es seit 2003 eine staatlich verordnete Pflichtzertifizierung für alle Brustzentren, deren Methoden sich stark an das ISO-Verfahren anlehnen. Die Mindestmengenanforderungen (150 Erstoperationen p.a für das Zentrum und 50 p.a. für den Operateur) haben zwischenzeitlich zu erheblichen Diskussionen geführt. Die Zertifizierung selbst erfolgt durch die Ärztekammer Westfalen-Lippe. Hervor zu heben ist bei dem NRW-Verfahren die Existenz von 25 Qualitätsindikatoren, die in den Qualitätsbericht eingehen und an Hand derer man unter anderem die Erfüllung der Anforderungen erkennen kann. 
Für das übrige Deutschland gilt - ebenfalls seit 2003 - das Zertifizierungsangebot der Deutschen Krebsgesellschaft und der Deutschen Gesellschaft für Senologie, dem sich bis Ende 2004 schon über 55 Brustzentren unterworfen haben. Das Verfahren besteht aus einer zwei-stufigen Zertifizierung: zunächst die Zertifizierung des Qualitätsmanagement vor allem nach ISO oder KTQ und anschließend eine Zertifizierung der onkologischen Anforderungen nach einer Begehung durch Fachexperten der OnkoCert (Einrichtung der Deutschen Krebsgesellschaft).

Auch die European Society of Mastology (EUSOMA) hat in 2003 Anforderungen an Brustzentren formuliert und über das europäische Parlament lanciert. Die weitergehenden Anforderungen der EUSOMA gegenüber dem Verfahren der beiden deutschen Fachgesellschaften bezüglich der Zahl der interdisziplinären Fallkonferenzen, der OP-Fallzahlen für Ersterkrankungen und der Spezialisierung der Operateure hat in Deutschland zu einiger Verunsicherung geführt.

\section{Externe Vergleiche der Leistungen}

gynäkologischer Abteilungen

Externe Vergleiche der medizinischen Versorgung durch einzelne Leistungserbringer haben in der Gynäkologie und Geburtshilfe in Deutschland, zunächst als freiwillige Perinatalerhebungen bzw. als „Qualitätssicherungsmaßnahme Operative Gynäkologie“ begonnen, eine lange Tradition. In Form der von der Bundesgeschäftsstelle Qualitätssicherung in Düsseldorf (BQS, www.bqs-online.de) im Auftrag des gemeinsamen Bundesausschusses betriebenen externen vergleichenden Qualitätssicherung sind diese Verfahren inzwischen von allen gynäkologischen/geburtshilflichen Abteilungen verpflichtend durchzuführen. Prinzipiell erhofft man sich von diesen externen Vergleichen, dass diejenigen Leistungserbringer, deren Versorgungsprozesse oder -ergebnisse außerhalb vorab definierter Referenzbereiche oder weit weg von der mittleren Ausprägung aller verglichenen Institutionen liegen, motiviert werden, ihre Versorgungsprozesse zu verändern.

Trotz der inzwischen über zwanzigjährigen Erfahrungen mit dieser Form interkollegial praktizierter und Institutionen übergreifender externer Vergleiche fehlt der streng wissenschaftliche Kausalitätsnachweis dafür, dass sie wirklich die Versorgung der Patientinnen verbessert haben. Die bisherigen Studien erlauben nur den Nachweis von Assoziationen, nicht aber den von Kausalbeziehungen. Dies gilt natürlich auch für die Interpretation von beobachteten Unterschieden zwischen Kliniken oder Abteilungen. Nichtsdestotrotz werden die unzweifelhaften Fortschritte bei der perinatalen Sterblichkeit und weiterer Versorgungsergebnisse, Veränderungen der Klinikstrukturen und die vielfachen Ablaufoptimierungen der Versorgungsprozesse auf die Existenz der externen Vergleiche zurückgeführt (z.B. Longo et al., 1998; Kazandjan \& Lied, 1998; Geraedts \& Selbmann, 1997; Geraedts et al., 1998).

Betrachtet man die vorgeschriebenen Inhalte für die Krankenhaus-Qualitätsberichte, die von den Landesverbänden der Krankenkassen ab 2005 ins Internet gestellt werden müssen und von den Krankenkassen und den Kassenärztlichen Vereinigungen für die Versicherten bzw. Kassenärzte vergleichend interpretiert werden können, dann überwiegen hier die Strukturmerkmale der Einrichtungen. Im so genannten Basisteil der Berichte geht es vor allem um Ausstattungs- und Leistungsmerkmale, die Personalqualifikation und die Erfüllung gesetzlich vorgeschriebener Qualitätssicherungsmaßnahmen. Im so genannten Systemteil sollen die Krankenhäuser vor allem beschreiben, welche Qualitätspolitik sie befolgen, wie Qualitätsmanagement intern umgesetzt wird und welche Qualitätsmanagement-Projekte durchgeführt wurden. Darüber hinaus kann über die Ergebnisse der externen QS-Maßnahmen oder über durchgeführte Selbst- oder Fremdbewertungen berichtet werden.

Wichtiger als eine Auflistung solcher struktureller Faktoren im Rahmen von externen Vergleichen ist jedoch, den Bürgern gegenüber darzustellen, ob sich ein Krankenhaus, ein Versorgungsnetzwerk, eine Praxisgemeinschaft oder eine einzelne Praxis an denjenigen diagnostischen und therapeutischen Verfahren orientiert, die sich als am besten wirksam herausgestellt haben. Hierfür sprechen u.a. Studienergebnisse von Chen und Mitarbeitern im Bereich der Herzinfarkttherapie, die zeigten, dass die konsequente Durchsetzung einer leitliniengerechten Behandlung der Faktor zu sein scheint, der diejenigen Krankenhäuser mit niedrigen Letalitätsraten von den Krankenhäusern mit höheren Raten unterscheidet (Chen et al., 1999).

Zur Vorbereitung valider externer Vergleiche zwischen Abteilungen oder Kliniken müssen die wissenschaftlichen medizinischen Fachgesellschaften Sorge tragen, dass methodisch einwandfreie Leitlinien für die wichtigsten Krankheitsbilder existieren und daraus Indikatoren für die Prozessqualität abgeleitet werden. Die Aktivitäten in dieser Richtung wurden in den letzten Jahren gerade auch in der operativen Gynäkologie verstärkt. Die im Rahmen der operativ-gynäkologischen, extern vergleichenden Qualitätssicherung verwendeten Indikatoren wurden als erste Qualitätsindikatoren der BQS auf ihre methodische Güte hin überprüft und wo nötig überarbeitet (Reiter et al., 2002), womit die Gynäkologie ihrer seit langem eingenommenen Vorreiterrolle in der deutschen Qualitätssicherung wieder einmal gerecht wurde.

Neben den erwähnten Prozessindikatoren sind auch patientenseitig ablesbare Ergebnisindikatoren zu vergleichen. Hierbei ist vor allem zu gewährleisten, dass die Vergleichbarkeit nicht durch die in der Regel unterschiedlichen Fallmixe der einzelnen Abteilungen oder Kliniken gestört wird. Die Glaubwürdigkeit von Vergleichsergebnissen steigt immens, wenn die Indikatoren für die Prozess- und Ergebnisqualität bei weitgehend vergleichbarem Fallmix analysiert werden. Dies kann z. B. dadurch geschehen, dass die zu vergleichenden Patientengruppen durch eine geeignet enge Wahl von Ein- und Ausschlusskriterien zwischen den Krankenhäusern vergleichbar gemacht werden. Will man aber die dadurch entstehende Verengung des Blickfeldes auf die Qualität der Versorgung nicht in Kauf nehmen, sind statistische Verfahren zur Standardisierung notwendig, z. B. in Form einer Fallmix-Adjustierung, bei denen der Fallmix der zu vergleichenden Abteilungen quasi rechnerisch vereinheitlicht wird.

Eine der wesentlichen Voraussetzungen für die Fallmix-Adjustierung besteht jedoch darin, dass die zur Adjustierung notwendigen Daten in ausreichender Qualität zur Verfügung stehen. Diese 
Bedingung ist derzeit nicht erfüllt. Noch nicht absehbar ist, ob die vollständige Umstellung der Krankenhausfinanzierung auf DRGs dazu führen wird, dass alle notwendigen Daten zur Fallmix-Adjustierung zumindest in den Krankenhäusern vorliegen werden. Zudem muss noch getestet werden, ob diese Daten für die Adjustierung von Qualitätsindikatorausprägungen verfügbar und auch wirklich geeignet sind.

Wenn versorgungsrelevante Indikatoren definiert und adjustiert sowie extern vergleichend analysiert wurden, kommt es schließlich darauf an, diese Analysen in einer Form zu präsentieren, die die verantwortlichen Ärztinnen und Ärzte zur Durchführung qualitätsfördernder Maßnahmen anregt. Hierzu muss die Darstellung der Qualitätsindikatoren so gestaltet werden, dass sie vom Betrachter

1. möglichst auf den ersten Blick durchschaut werden,

2. die Tendenz zur Orientierung am Mittelmaß durch eine Orientierung an den jeweils Besten ersetzen und

3. zur Qualitätsverbesserung motivieren.

Diese Prinzipien wurden bei der Entwicklung der so genannten Qualitätsmonitore für die operative Gynäkologie verfolgt (Geraedts 1997). Bei der Übertragung und Vereinheitlichung des Verfahrens durch die BQS hat sich jedoch eine andere Form der Darstellung durchgesetzt, deren Lesbarkeit und Auswirkungen auf die Motivation von Qualitätsverbesserungen noch zu untersuchen wäre.

\section{Patienteninformierung}

Damit externe Vergleiche aber nicht nur von der Fachöffentlichkeit, sondern auch von (potenziellen) Patienten - und anderen „Kunden“ der Kliniken bzw. Ärzte - verstanden und verwendet werden, sind besondere Anstrengungen bei der inhaltlichen und formalen Gestaltung der Vergleiche notwendig.

Bisher scheinen die Patienten und „Einkäufer“ von Versorgungsnetzwerk-Dienstleistungen wenig Gebrauch von externen Vergleichen zu machen. Darauf deuten U.S.-amerikanische Erfahrungen hin: Schneider und Epstein berichten über eine telefonische Befragung von Patienten, die sich 1995 im Staat Pennsylvania einem koronarchirurgischen Eingriff unterzogen hatten (Schneider und Epstein, 1998). Hier werden seit 1992 die Mortalitätsraten jedes Herzchirurgen und jeder herzchirurgischen Klinik jährlich in einem Konsumentenführer in hoher Auflage veröffentlicht. Jedoch kannten nur 12\% der Patienten vor ihrem Eingriff diese Veröffentlichung. Weniger als $1 \%$ der Patienten kannte die genaue Mortalitätsrate und sagte, die Kenntnis habe einen mittleren bis hohen Einfluss auf ihre Wahl des Arztes bzw. Krankenhauses gehabt.

Eine Befragungsstudie von „Einkäufern“ von Versorgungsleistungen ergab sogar, dass gerade die Informationen zur Akkreditierung der Leistungserbringer und zur Qualität der Versorgung, wie sie in den USA bisher veröffentlicht werden, als am wenigsten relevant für die Einkaufsentscheidung bewertet wurden (Legnini et al., 2000). Wichtigstes Kriterium bei der Wahl eines Leistungsanbieters war dagegen der Preis, ein Kriterium, das in Deutschland wegen des Sachleistungsprinzips allerdings keine
Rolle spielt. Die Autoren schließen daraus, dass die bisherigen Patienten- und Kundeninformationen stark überarbeitet und die Verbraucher in der Nutzung dieser Informationen trainiert werden müssen, damit die Qualität der Versorgung auch tatsächlich zum Wettbewerbsfaktor werden kann.

Einer Studie von Robinson und Brodie folgend vertrauen die Patienten bei Informationen zur Qualität einzelner Leistungserbringer bislang am meisten auf das Urteil von Freunden und Bekannten (50\%) (Robinson und Brodie, 1997). Danach folgen Ergebnisse aus Patientenbefragungen (34\%). Weniger als ein Drittel der Patienten nutzen Ärzte, Versicherungen oder Regierungsorganisationen bzw. Beratungsstellen als Informationsquelle. Bei der Auswahl von Kliniken spielen die Familie und die betreuenden Ärzte mit jeweils rund 60\% eine gleich wichtige Rolle, gefolgt von Patientenbefragungen (43\%). Die übrigen Informationsquellen, unter anderem externe Vergleichslisten von „offiziellen“ Stellen oder Zeitschriften, werden zu jeweils weniger als $25 \%$ genutzt.

Für die neu im Sozialgesetzbuch ( $§ 65$ b SGB V) verankerten, von den Krankenkassen zu fördernden Organisationen zur Verbraucher- und Patientenberatung wird es also viel zu tun geben, um sich als anerkannte Informationsquellen zu etablieren. Zunächst scheint es dem Gesetzgeber aber nicht darum zu gehen, den Patienten mithilfe dieser Organisationen Informationen zur Qualität der Versorgung durch einzelne Ärzte oder Kliniken an die Hand zu geben. Vielmehr stehen allgemeine Gesundheitsinformationen im Vordergrund des Interesses. Damit Patienten sich ein Bild über die Qualität dieser Informationen schaffen können, koordinierte das Bundesministerium für Gesundheit und Soziale Sicherung die Gründung eines „Aktionsforum Gesundheitsinformationssystem für Deutschland (AFGIS) e.V.“. Sein Ziel ist es, ein Netzwerk von Organisationen aufzubauen, auf deren Gesundheitsinformationen sich die Patienten verlassen können, und dieses auch durch ein Logo erkenntlich zu machen.

Langfristig könnte dieses Netzwerk auch dazu genutzt werden, den Patienten nützliche Informationen zur Auswahl von Ärzten und Kliniken nach Qualitätsgesichtspunkten zu vermitteln. Um auch bei dieser Entwicklung Einfluss dahingehend auszuüben, dass keine Fehlinformationen verbreitet werden, würde es sich lohnen, konstruktiv am Aufbau des Patienteninformationsnetzwerks teilzunehmen.

\section{Ausblick}

Qualitätssicherung in der operativen Gynäkologie heißt nicht, monoman das Konzept der externen Vergleiche zu verfolgen. Vielmehr steht eine Reihe innovativer qualitätssichernder Ansätze zur Verfügung, von denen hier einige besprochen wurden. Um diese Ansätze in der operativen Gynäkologie zur Anwendungsreife zu bringen, bedarf es des intensiven Engagements vieler im Qualitätsmanagement erfahrener Gynäkologen und der Fachgesellschaften. Die Vielzahl bereits existierender Aktivitäten zum Qualitätsmanagement lässt darauf hoffen, dass auch die neueren Entwicklungen von den Gynäkologen wahrgenommen und aktiv mit gestaltet werden. 


\section{Literatur}

${ }^{1}$ Blum K, Hanel E, Mündermann-Hahn A, Storm T, Thoma E. Leitfaden: Klinisches Audit. Band 143 der Schriftenreihe des Bundesministeriums für Gesundheit. Baden-Baden: Nomos Verlagsgesellschaft, 2002

${ }^{2}$ Chen J, Radford MJ, Wang Y, Marciniak TA, Krumholz HM. Do "America's Best Hospitals" perform better for acute myocardial infarction? NEJM 1999; 340: 286-292

${ }^{3}$ Eichhorn S, Koester H, Selbmann HK. Qualitätssicherung in der operativen Gynäkologie. Materialien und Berichte der Robert-Bosch-Stiftung Nr. 31. Gerlingen: Bleicher, 1989

${ }^{4}$ Geraedts M, Selbmann HK. Erfahrungen mit externer Qualitätssicherung im gynäkologisch operativen Bereich. Gynäkol Geburtshilfliche Rundsch 1997; 37: $14-20$

${ }^{5}$ Geraedts M. Operative Gynäkologie. In: Scheibe O (Hrsg). Qualitätsmanagement in der Medizin. Landsberg: Ecomed 1997: IV-2.2.2.1: $1-13$

${ }^{6}$ Geraedts M, Koester H, Berg D, Rauskolb R, Scheidel P, Selbmann HK. Qualitätssicherung in der operativen Gynäkologie. Band 98 der Schriftenreihe des Bundesministeriums für Gesundheit. Bundesministerium für Gesundheit (Hrsg). Baden-Baden: Nomos, 1998

7 Glück D, Selbmann HK. Genuin-medizinische Zertifizierungsverfahren in ausländischen Krankenhäusern. Z ärztl Fortbild Qualitätssich 2000; 94/10: 659-664
${ }^{8}$ Kazandjan VA, Lied TR. Cesarean section rates: Effects of participation in a performance measurement project. Jt Comm J Qual Improv 1998; 24/4: 187-196

${ }^{9}$ Legnini MW, Rosenberg LE, Perry MJ, Robertson HJ. Where does performance measurement go from here? Health Affairs 2000; 19/3: $173-177$

${ }^{10}$ Longo DR, Land G, Schramm W, Fraas J, Hoskins B, Howell V. Consumer reports in health care. Do they make a difference in patient care? JAMA 1997; 278: 1579-1584

11 National Committee for Quality Assurance (NCQA): Standards for Accreditation of Managed Care Organizations 1998. Washington, DC, NCQA, 1998

${ }^{12}$ Reiter A, Geraedts M, Stillger R, Misselwitz B. Evaluation und Überarbeitung der Qualitätsindikatoren in der operativen Gynäkologie. Geburtsh Frauenheilkunde 2002; 62: 635-643

${ }^{13}$ Robinson S, Brodie M. Understanding the Quality challenge for health consumers: The Kaiser/AHCPR Survey. Jt Comm J Qual Impr. 1997; 23/ 5: $239-244$

${ }^{14}$ Schneider EC, Epstein AM. Use of public performance reports. JAMA 1998; 279/20: $1638-1642$

${ }^{15}$ Weert C. van. Developments in professional quality assurance towards quality improvement: Some examples of peer review in the Netherlands and the United Kingdom. Internat J Quality in Health 2000; $12 / 3: 239-242$ 